CERN-EP-2000-060

May 9, 2000

\title{
THE COMPACT EMULSION SPECTROMETER
}

S. Buontempo ${ }^{1)}$, L. Camilleri' ${ }^{2}$, M.G. Catanesi ${ }^{3)}$, M. Chizov $^{2)}$, A. De Santo ${ }^{2,10)}$, E. Do Couto e Silva ${ }^{2}$, M. Doucet ${ }^{2)}$, J. Goldberg ${ }^{4)}$, G. Grégoire ${ }^{5)}$, G. Grella ${ }^{6)}$, L. Linssen ${ }^{2)}$, B. Lisowski ${ }^{2)}$, M. Litmaath ${ }^{2)}$, J. Kokkonen ${ }^{2)}$, O. Melzer ${ }^{7)}$, V. Mexner ${ }^{2)}$, P. Migliozzi ${ }^{1)}$, M.T. Muciaccia ${ }^{3)}$, E. Niu ${ }^{2)}$, J. Panman ${ }^{2)}$, I.M. Papadopoulos ${ }^{2)}$, E. Pesen ${ }^{7)}$, E. Radicioni ${ }^{3)}$, S. Ricciardi ${ }^{2,9, *)}$, O. Runolfsson ${ }^{2)}$, S. Simone ${ }^{3)}$, F.J.P. Soler ${ }^{2, a)}$, U. Stiegler ${ }^{2)}$, J.W.E. Uiterwijk ${ }^{7)}$, B. Van De Vyver ${ }^{8)}$

(To be submitted to Nuclear Instruments and Methods)

\begin{abstract}
An emulsion spectrometer has been built and tested with pion beams in a $0.7 \mathrm{~T}$ magnetic field. A momentum resolution, $\Delta \mathrm{P} / \mathrm{P}=13 \% \pm 1 \%$, has been obtained for 5 and $10 \mathrm{GeV} / \mathrm{c}$ particles.
\end{abstract}

\footnotetext{
*) Corresponding author.

1) Università di Napoli and INFN, Napoli, Italy.

2) CERN, Geneva, Switzerland.

3) Università di Bari and INFN, Bari, Italy.

4) Technion, Haifa, Israel.

5) Université Catholique de Louvain, Louvain-la-Neuve, Belgium.

6) Università di Salerno and INFN, Salerno, Italy.

7) NIKHEF, Amsterdam, The Netherlands.

8) Inter-University Institute for High Energies (ULB-VUB), Brussels, Belgium.

9) Now at Royal Holloway and Bedford New College, University of London, Egham, Surrey, U.K.

10) Now at University of Oxford, Oxford, U.K.

a) Supported by a TMR fellowship from the European Commission.
} 


\section{Introduction}

Many particle physics experiments face space constraints and a limited extension of the magnetic volume. The possibility of measuring momenta over short distances is therefore of great value. An emulsion spectrometer is a sandwich construction of nuclear emulsion plates and light-weight spacers placed in a magnetic field. Given the sub-micron precision of nuclear emulsions, the momentum can be accurately determined despite having short lever arms or being in a moderate magnetic field. An example of such an application is the charge determination of particles with a momentum of a few $\mathrm{GeV}$ within a large solid angle in a fixed target experiment.

Previous tests of a similar emulsion spectrometer in an intense magnetic field (2 T) were performed for heavy ion studies, for its ability to work in high track density environments [1]. However, its characteristics can be successfully exploited in high energy neutrino experiments where the low interaction rate allows a long lasting exposure of emulsions (up to several years as has been the case in CHORUS [2]). For instance, a possible design for a next generation $\nu_{\tau}$ appearance oscillation experiment, TOSCA [3], includes a compact emulsion spectrometer in each module to interface conventional tracking chambers and a silicon detector and to improve acceptance for low momentum tracks.

We have designed a device able to determine the momentum of charged tracks in the range 1 to $20 \mathrm{GeV} / \mathrm{c}$, which is of interest for high energy neutrino interactions. This detector can work in a magnetic field of $0.7 \mathrm{~T}$, as is envisaged for TOSCA. A prototype has been built and tested using a pion beam at CERN. The main features of the design are described in Section 2. Section 3 summarises the experimental conditions of data taking. In Section 4 the details of the analysis are reported. The results can be found in Section 5 .

\section{Design of a small scale prototype}

The emulsion spectrometer in the test consists of an alternating arrangement of 8 emulsion sheets interspersed with 7 light plastic foam $\left(\right.$ Rohacell $\left.^{\circledR}\right)$ spacers placed in a honeycomb box. The active plates consist of two $100 \mu \mathrm{m}$ emulsion layers coated on either side of an $800 \mu \mathrm{m}$ plastic base. They are individually vacuum packed in light-tight paper. The separator and box materials are chosen to minimise the mass density in order to reduce multiple scattering. The spacers are coated with a $127 \mu \mathrm{m}$ layer of fibre glass to ensure sufficient flatness, thickness uniformity and mechanical rigidity. The flatness is required to be better than $\pm 20 \mu \mathrm{m}$ over an area of about $200 \times 200 \mathrm{~mm}^{2}$. The distance between successive emulsion plates increases in the beam direction from $8 \mathrm{~mm}$ to $32 \mathrm{~mm}$, as shown in Fig. 1. For the purpose of the test the transverse size of the emulsion plates is limited to $180 \times 180 \mathrm{~mm}^{2}$.

In each spacer and in the upstream face of the honeycomb box four holes were drilled to host bi-directional X-ray guns, used as a reference in the scanning. The accuracy of this reference depends on the size, the shape regularity and the sharpness of the black circular spot that they produce in the emulsions, the typical diameter being about $1 \mathrm{~mm}$. A concentrated liquid solution, of about $1 \mathrm{MBq} / \mathrm{cm}^{3}$ activity, of ${ }^{55} \mathrm{Fe}$ was used. The high intensity of the source compensated for the short duration of the test.

\section{$3 \quad$ The test beam setup and data taking}

The characteristics of the emulsion spectrometer were studied using negative pion beams from the CERN PS X9 beam line during September 1997. Three identical spectrometers were used in different tests: 

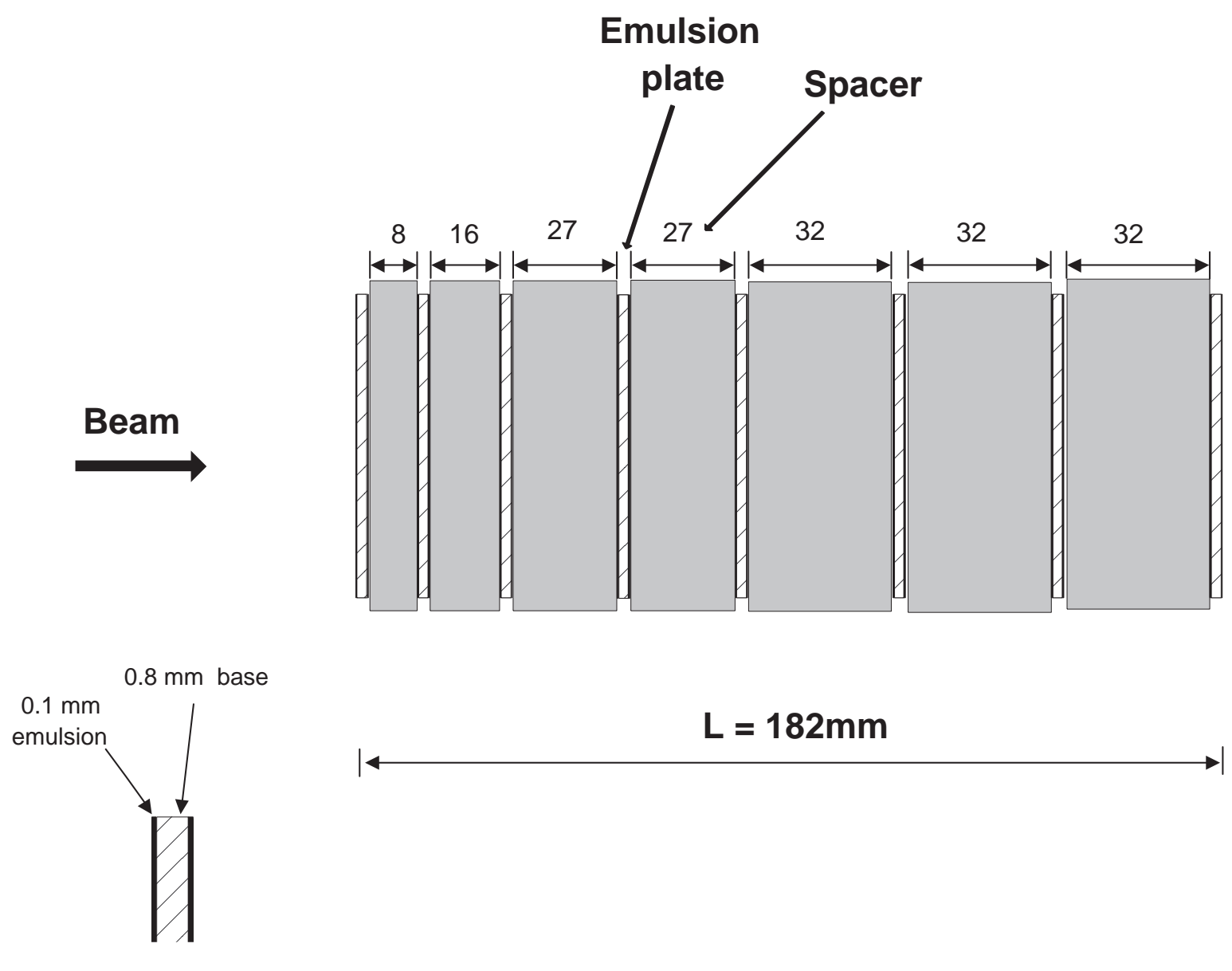

\section{Emulsion plate}

Figure 1: Side view of the emulsion spectrometer used in the test. The thickness of the emulsion is not drawn to scale. Units are mm. 
- $15 \mathrm{GeV} / \mathrm{c}$ pions and tracks from pion interactions in an upstream, $90 \mathrm{~mm}$ thick, emulsion target;

- 5 and $10 \mathrm{GeV} / \mathrm{c}$ beams without upstream target;

- 3 and $5 \mathrm{GeV} / \mathrm{c}$ beams and an upstream target for the measurement of the white kink cross-section, that is the process of pion scattering in emulsion without visible activity at the vertex.

The results reported here refer to the dedicated exposure to 5 and $10 \mathrm{GeV} / \mathrm{c}$ negative pion beams. The momentum was accurate to better than $\pm 1 \%$ and the beam spot was wide enough to obtain a uniform particle density in the fiducial area. Beam profiles and intensities were monitored using delay wire chambers and a scintillation counter telescope.

The trigger was given by the coincidence of three scintillation counters placed along the beam line, upstream of the detector. The last counter, made up of a $50 \times 50 \mathrm{~mm}^{2}$ scintillating tile, was placed inside the magnetic field, at about $100 \mathrm{~mm}$ from the spectrometer. This defined the fiducial area. About 10,000 triggers were counted in the 5 $\mathrm{GeV} / \mathrm{c}$ run and 9,000 in the $10 \mathrm{GeV} / \mathrm{c}$ run, corresponding to a particle density of about 8 $\mathrm{mm}^{-2}$. The total duration of the exposure was half an hour for the first and an hour for the second run.

The spectrometer was placed close to the centre of the $1 \times 1 \mathrm{~m}^{2}$ poles in the $50 \mathrm{~cm}$ gap of a C-magnet, in a region where the field uniformity, $\Delta \mathrm{B} / \mathrm{B}$, is better than $8 \cdot 10^{-3}$. The magnetic field strength was set to $0.7 \mathrm{~T}$ and its direction was reversed between the two runs to obtain a clear separation between the two samples after scanning.

The emulsion plates were scanned at CERN with an automatic scanning system based on a computer-driven optical microscope. The system is described in [4]. The typical time required to digitise all the tracks, inside a cone with an opening angle of $\pm 40 \mathrm{mrad}$, in the central $10 \times 10 \mathrm{~mm}^{2}$ of an emulsion plate, is 4 hours. Track reconstruction is performed offline using the position and slope of all the segments found in each plate.

\section{$4 \quad$ Data Analysis}

The result of the scanning is a list of the position and the slope for all track segments. The coordinate system is chosen such that $X$ is parallel to the magnetic field and $Z$ is the longitudinal coordinate. The initial position of the plates is known with a precision of 0.1 to $1 \mathrm{~mm}$. They are given by measurements with a calliper of the X-ray gun holes and of the thickness of each spacer. Another source of error is the determination of the centre of the X-ray spot on the emulsion plate during the scanning. The effect is particularly severe for those cases where the contour of the spot is not sharply defined. In addition, the spacers were not exactly parallel to each other inside the box. This effect will be referred to as a tilt.

A final calibration to better than 10 (100) micron in the transverse (longitudinal) coordinates is necessary to achieve a good resolution for this short lever arm spectrometer. It is obtained by iterating different steps: plate-to-plate calibration, track finding and track fitting.

As the magnetic field was never turned off during the data taking, no straight tracks are recorded in the emulsion. Therefore we have been forced to use the knowledge of the nominal beam momentum to correct for tilt beforehand. The two momentum samples can be separated by a selection on the slope in the bending projection in the first plate. With our choice of axes the slope is negative for $5 \mathrm{GeV} / \mathrm{c}$ particles and positive for $10 \mathrm{GeV} / \mathrm{c}$ particles. The uncorrected angular distribution in the bending projection of all segments is shown in Fig. 2. 


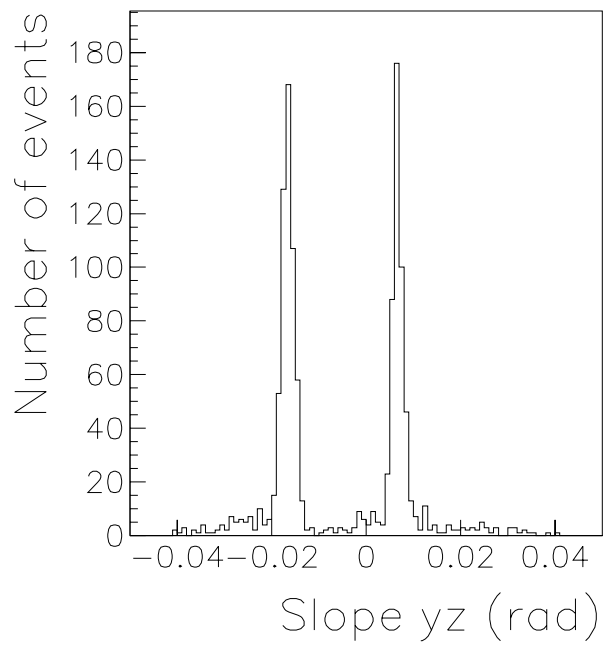

Figure 2: Angular distribution of the track segments in the bending projection of the first plate.

\subsection{Calibration}

A Gaussian fit to the uncorrected angular distributions of the segments in each plate has been performed to compute the tilt corrections, defined as the differences between the peak value for plate $n$ and for the first plate. For the bending plane the expected deviations due to the magnetic field are subtracted. The corrections are of the order of a few mrad and, as expected, approximately equal for the negative and positive slope samples. Raw data have been corrected using the positive slope sample since this provides the straightest tracks. We have further assumed that the first plate, the one most constrained by mechanical construction, coincides with the plane orthogonal to the $Z$ direction.

In addition, 6 parameters are determined by a plate to plate calibration based on a minimisation procedure. The parameters are:

-3 translation offsets;

- 2 shrinkage parameters or scale factors for the emulsion plate;

- one rotation angle around the $Z$ axis.

The procedure consists in matching maps of points belonging to two subsequent plates and it has been adapted from the one used by CHORUS [2], which operated without magnetic field. Segments are linearly extrapolated to a virtual plane at half the distance between the plates to be calibrated, to reduce the magnetic field effects. For each plate, the centre of the map is determined by the centre of gravity of the position of the segments. The total distance between each point belonging to the most upstream map and the closest one belonging to the second map is minimized using the downhill simplex method [5]. With the first plate taken as a reference, the calibration constants had to be propagated plate by plate. Unavoidably, the last plates, which are further apart, suffer from a bigger total calibration error. Statistical errors are evaluated by repeating the calibration several times. They range from a few microns in the transverse coordinates to hundreds of microns in the longitudinal one. Being poorly determined by the inter-calibration procedure, the longitudinal position of the plates was not corrected for in the subsequent steps of the analysis. This uncertainty introduces an error in the absolute momentum determination 
of a few hundred $\mathrm{MeV}$. In a different experimental environment this aspect could be easily cured, for instance by recording two samples of straight reference tracks impinging at big angles w.r.t. the longitudinal axis.

\subsection{Track finding}

The accuracy of the first plate-to-plate calibration is sufficient to join segments that belong to different plates starting from plate 1 and using a first or second order polynomial extrapolation in the non-bending and bending projections, respectively. The coefficients of the polynomial are determined by interpolation of the points which are symmetricallypositioned around the interpolation argument. A chi-squared is built using position and slope information of the extrapolated track from plate $1,2, \ldots \mathrm{j}$ and any segment of plate $j+1$. The segment corresponding to the smallest $\chi^{2}$ is added to the track provided the $\chi^{2}$ does not exceed an upper limit. This criterion was tuned on the data. The tracking algorithm allows for one missing plate and, when there are no segments satisfying the condition on the $\chi^{2}$, an extrapolation to the subsequent plate is tried. The procedure does not require sophisticated algorithms to reduce the amount of computation since the number of segments is limited to about 1000 per plate.

\subsection{Track fitting}

In the absence of absorbing material, the trajectory of a charged particle in a constant magnetic field is a helix of constant curvature. The magnetic field goes along the $X$ axis of the reference system. The trajectory is well approximated by a circle sector in the $Y Z$ plane and the displacement in $X$ is proportional to the length of arc $S$ that is described in $Y Z$.

The circumference fit is performed using the linear approach of [6]:

$$
\delta_{k}=\delta+\left(y_{k}-y_{1}\right) \sin \phi_{0}-\left(z_{k}-z_{1}\right) \cos \phi_{0}-\frac{C}{2(1-C \delta)}\left(\left(y_{k}-y_{1}\right)^{2}+\left(z_{k}-z_{1}\right)^{2}\right),
$$

where $\left(x_{k}, y_{k}, z_{k}\right)$ are the coordinates of the $k$ 'th point and $\delta_{k}$ is its distance to the circumference. The point $\left(x_{1}, y_{1}, z_{1}\right)$ is chosen as reference and the 3 parameters are the curvature of the track, $\mathrm{C}$, the azimuthal angle of the momentum vector at the position of closest approach to the reference point, $\phi_{0}$ and $\delta$, the distance of closest approach to the reference point.

The $\chi^{2}$ to be minimized is

$$
\chi^{2}=\Sigma_{k j} w_{k j} \delta_{k} \delta_{j}+\Sigma_{k j} w_{k j}^{\prime}\left(x_{k}-x_{0}-s_{k} \tan \lambda\right)\left(x_{j}-x_{0}-s_{j} \tan \lambda\right),
$$

where $s_{k}$ is the arc length of the $k$-th point. Two additional fit parameters are $\lambda$, related to the polar angle of the momentum vector $\left(\tan \lambda=1 / \tan \theta, \mathrm{P}_{x}=\mathrm{P} \cos \theta\right)$ and $x_{0}$, which is the $X$ position where the particle is at the distance of closest approach in the $Y Z$ plane.

The weights are approximated by the elements of the inverse of the two covariance matrices between the hits in the $Y Z\left(w_{k j}\right)$ and the $X Z\left(w_{k j}^{\prime}\right)$ planes. The covariance matrices are described in the following Section.

The angular information for each segment is used in the fit to retrieve the coordinates of the two points that correspond to the two measurements on opposite sides of the emulsion base. A track which spans $n$ plates will correspond to $2 n$ points and its trajectory will be determined by a fit with $2 n-5$ degrees of freedom. The correlation between all these pairs of hits is taken into account in the error matrix. 


\subsection{Errors}

The $2 n \times 2 n$ covariance matrices have to account for different sources of errors associated with the individual measurements.

Three independent categories can be distinguished: intrinsic, calibration and multiple scattering errors. Intrinsic errors arise from the finite resolution of the nuclear emulsion, they include also all systematics associated to the scanning system. They contribute both to diagonal and non-diagonal elements. The non-diagonal elements take into account the correlation between measurements on either side of the same plate. Calibration errors are those related to the internal alignment procedure and to the reconstruction of the original position in the magnet. They are of the order of $10 \mu \mathrm{m}$.

The multiple scattering matrix has been computed in the approximation of straight tracks and considering the density and radiation length of the materials as reported in Table 1 . The values of diagonal elements increase as a function of the plate number from 0 to $100 \mu \mathrm{m}$.

\begin{tabular}{|l|c|c|c|}
\hline Material & Thickness & Radiation length $(\mathrm{cm})$ & Density $\left(\mathrm{g} / \mathrm{cm}^{3}\right)$ \\
\hline Emulsion & $16 \times 100 \mu \mathrm{m}$ & 2.97 & 3.7 \\
\hline Plastic base & $8 \times 800 \mu \mathrm{m}$ & 42 & 1.032 \\
\hline Fibre glass & $14 \times 127 \mu \mathrm{m}$ & 10.56 & 2.56 \\
\hline Rohacell $\left.^{\mathrm{R}}\right)$ & $8 \div 32 \mathrm{~mm}$ & 676 & 0.06 \\
\hline
\end{tabular}

Table 1: Material thicknesses and properties.

\section{$5 \quad$ Results}

The distributions of the residuals, defined as the difference between the hit position corresponding to the best fit and the measured hit position, are, within errors, centered at zero.

\begin{tabular}{|l||c|c||c|c|}
\hline \hline & Sample slope - & $\epsilon(\%)$ & Sample slope + & $\epsilon(\%)$ \\
\hline all segments in plate 1 & 548 & & 446 & \\
tracks spanning 8 plates & 339 & 62 & 292 & 65 \\
\hline$\chi^{2}>0.01$ & 248 & 45 & 240 & 54 \\
\hline \hline
\end{tabular}

Table 2: Number of tracks found and efficiencies in the two different samples.

The initial number of segments and the number of track candidates obtained when requiring segments up to the last plate are reported in Table 2. The efficiencies, $\epsilon$, for track finding and fitting before and after a selection on $\chi^{2}$ are also shown. They are the product of scanning, track finding and fitting efficiencies. It should be noted that a scanning efficiency of $82 \%$ for each single plate could by itself account for the results, as can be computed by taking into account that the tracking procedure allows to skip one plate if the segment is not found.

The distribution of the inverse of the momentum shows two peaks, corresponding to opposite slopes of the track segment as measured in the first plate. In figure 3, only long tracks, that is followed up to plate 8, enter into the histogram. Tracks are further selected 

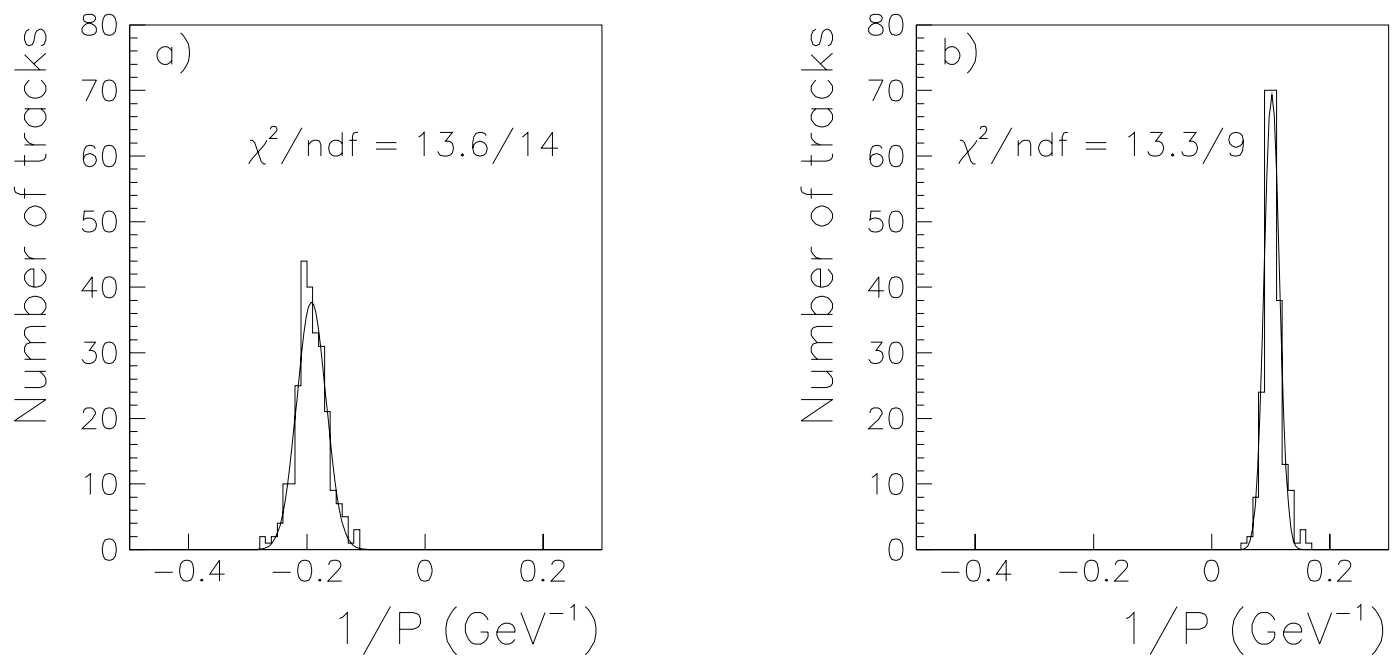

Figure 3: Distributions of the inverse of the momentum for tracks reconstructed in all plates: $5 \mathrm{Gev} / \mathrm{c}$ sample (a); $10 \mathrm{GeV} / \mathrm{c}$ sample (b).

requiring a $\chi^{2}$ probability higher than $10^{-2}$. The central values of the inverse momentum, as obtained by a gaussian fit, are $-0.192 \pm 0.002 \mathrm{GeV}^{-1}$ and $0.102 \pm 0.001 \mathrm{GeV}^{-1}$. The one sigma resolution on the momentum is $1.3 \pm 0.1 \mathrm{GeV} / \mathrm{c}$ for $10 \mathrm{GeV} / \mathrm{c}$ pions and 0.65 $\pm 0.05 \mathrm{GeV} / \mathrm{c}$ for $5 \mathrm{GeV} / \mathrm{c}$, which corresponds to an accuracy of

$$
\frac{\Delta \mathrm{P}}{\mathrm{P}}=13 \% \pm 1 \%
$$

This value is independent of the momentum, as expected, since the multiple scattering errors dominate. The expected momentum resolution from a detailed Monte Carlo simulation agrees with the experimental value, and predicts $\frac{\Delta \mathrm{P}}{\mathrm{P}}<15 \%$ for charged particles up to $20 \mathrm{GeV} / \mathrm{c}$.

\section{Conclusion and discussion}

We have exposed a compact emulsion spectrometer to pion beams of $5 \mathrm{GeV} / \mathrm{c}$ and $10 \mathrm{GeV} / \mathrm{c}$. The detector was optimised for the momentum measurement of particles from high energy neutrino interactions (order of magnitude of $10 \mathrm{GeV} / \mathrm{c}$ ) over a short lever arm and in a magnetic field smaller than $1 \mathrm{~T}$.

The device can also be used as a tracking interface between an emulsion target and a conventional large volume tracker. Since automatic scanning systems can be very fast, tracking speed is not an issue and a matching procedure using maps based on full area scanning has been successfully tried. The tracking efficiency loss can be overcome by an improved quality check of the scanning and more sophisticated tracking algorithms.

The experimental resolution for momentum measurement, $\Delta \mathrm{P} / \mathrm{P}$, is $13 \% \pm 1 \%$ and agree with Monte Carlo simulations. This method can therefore be used to measure the momentum and the charge sign of traversing particles. The alignment procedure can be simplified by recording in the emulsions the tracks of muons of momenta above $10 \mathrm{GeV} / \mathrm{c}$, without magnetic field, impinging on the spectrometer at large angles. 


\section{$7 \quad$ Acknowledgements}

We wish to express our deep appreciation to the engineers and technical collaborators who made this test possible. In particular we wish to thank: C. Rosset, for the design of the support structure, P. Schilly and his group for the construction and installation, R. Rey-Mermier and F. Bergsma for the magnetic field measurements. We are also indebted to the PS staff and in particular to O. Ferrando for the technical support and the beam studies.

\section{References}

[1] T. Hayasino et al., Nucl. Instrum. and Meth. A271 (1988) 518-522 and A255 (1987) 482-485.

[2] E. Eskut et al., Nucl. Instrum. and Meth. A401 (1997) 7.

[3] A.S. Ayan et al., CERN-SPSC-97-05, CERN/SPSC I213, March 1997.

[4] I.M. Papadopoulos,"Nuclear Emulsion Readout Techniques developed for the Chorus Experiment", IEEE Nuclear Science Symposium and Medical Imaging Conference, Seattle, Oct 26-30, 1999.

[5] W.H. Press et al.," Numerical Recipes in C", 2nd edition, p.480, Cambridge University Press, 1993.

[6] V. Karimäki, Comput. Phys. Commun. 69 (1992) 133-41. 